\title{
Maladaptive mood repair, atypical respiratory sinus arrhythmia, and risk of a recurrent major depressive episode among adolescents with prior major depression
}

\author{
M. Kovacs ${ }^{1 *}$, I. Yaroslavsky ${ }^{2}$, J. Rottenberg ${ }^{3}$, C. J. George ${ }^{4}$, E. Kiss ${ }^{5}$, K. Halas ${ }^{5}$, R. Dochnal ${ }^{5}$, I. Benák ${ }^{5}$, \\ I. Baji ${ }^{5}$, A. Vetró ${ }^{5}$, A. Makai $^{5}$ and K. Kapornai ${ }^{5}$ \\ ${ }^{1}$ University of Pittsburgh School of Medicine, Pittsburgh, PA, USA \\ ${ }^{2}$ Cleveland State University, OH, USA \\ ${ }^{3}$ University of South Florida, FL, USA \\ ${ }^{4}$ University of Pittsburgh Medical Center, PA, USA \\ ${ }^{5}$ Szeged University, Hungary
}

Background. Because depressive illness is recurrent, recurrence prevention should be a mainstay for reducing its burden on society. One way to reach this goal is to identify malleable risk factors. The ability to attenuate sadness/dysphoria (mood repair) and parasympathetic nervous system functioning, indexed as respiratory sinus arrhythmia (RSA), are impaired during depression and after it has remitted. The present study therefore tested the hypothesis that these two constructs also may mirror risk factors for a recurrent major depressive episode (MDE).

\begin{abstract}
Method. At time 1 (T1), 178 adolescents, whose last MDE had remitted, and their parents, reported on depression and mood repair; youths' RSA at rest and in response to sad mood induction also were assessed. MDE recurrence was monitored until time 2 (T2) up to 2 years later. Mood repair at T1 (modeled as a latent construct), and resting RSA and RSA response to sadness induction (RSA profile), served to predict onset of first recurrent MDE by T2.
\end{abstract}

Results. Consistent with expectations, maladaptive mood repair predicted recurrent MDE, above and beyond T1 depression symptoms. Further, atypical RSA profiles at T1 were associated with high levels of maladaptive mood repair, which, in turn, predicted increased risk of recurrent MDE. Thus, maladaptive mood repair mediated the effects of atypical RSA on risk of MDE recurrence.

Conclusions. This study documented that a combination of behavioral and physiological risk factors predicted MDE recurrence in a previously clinically referred sample of adolescents with depression histories. Because mood repair and RSA are malleable, both could be targeted for modification to reduce the risk of recurrent depression in youths.

Received 20 October 2015; Revised 23 February 2016; Accepted 24 February 2016; First published online 20 May 2016

Key words: Adolescents, emotion regulation, mood repair, recurrent major depression, respiratory sinus arrhythmia.

\section{Introduction}

The recurrent nature of depressive illness has been one of the most extensively replicated finding in the psychopathological literature (for reviews, see Burcusa \& Iacono, 2007; Hardeveld et al. 2010). For example, within 5 years of having recovered from an episode of (usually) major depression, up to $85 \%$ of clinically referred adults had another depression episode (Mueller et al. 1999; Solomon et al. 2000). High rates of recurrence characterize clinically depressed youths as well (for a

* Address for correspondence: M. Kovacs, Ph.D., University of Pittsburgh School of Medicine, WPIC, 3811 O'Hara Street, Pittsburgh, PA 15213, USA.

(Email: kovacs@pitt.edu) review, see Rao \& Chen, 2009). Although major depression may be somewhat less recurrent in samples outside of specialized mental health care settings (e.g. Hardeveld et al. 2013), prevention of recurrence is critical for reducing the overall burden of depression on society.

One approach to recurrence prevention is to identify and then ameliorate contributory factors (e.g. Farb et al. 2015). However, reliable predictors of depression recurrence, such as number of prior episodes or stressful life events (e.g. Hardeveld et al. 2013; Harkness et al. 2014), which were consistently identified in earlier studies, have had limited implications for prevention efforts. Thus, the research emphasis has been shifting to the study of risk factors that are malleable, especially those that might link behavioral and 
physiological aspects of vulnerability (Marchetti et al. 2012; Cuthbert \& Insel, 2013; Farb et al. 2015).

One important area of individual differences relevant to depression risk is the ability to appropriately self-regulate sadness and dysphoria (mood repair), which is impaired in the context of major psychopathology (Aldao et al. 2010; Gross, 2013). Consistent with the typical characterization of depressive disorders (American Psychiatric Association, 2000), clinically depressed individuals usually report finding it difficult (or impossible) to attenuate their sad mood. More importantly, mood repair problems persist after recovery from depression and usually involve excessive use of responses, such as rumination, that exacerbate or prolong rather than attenuate dysphoria (Ehring et al. 2008; Kovacs et al. 2009; Kanske et al. 2012). The persistence of problematic mood repair may increase the risk of depression recurrence by exacerbating and prolonging normally occurring sad affect, which then can lead to a cascade of further depression symptoms.

There is considerable evidence that problematic mood repair prospectively predicts depression symptoms. In their classic study, Nolen-Hoeksema \& Morrow (1991) found that individuals' pre-existing ruminative style of coping with sadness predicted severity and duration of depression symptoms after experiencing a natural disaster. We found that, controlling for depression levels, the habitual use of maladaptive mood repair (MMR) responses predicted depression symptoms about 1 year later among adults who varied in depression risk (Kovacs et al. 2009). Conversely, competent emotion regulation in a community sample of adults predicted lower levels of depression symptoms 5 years later (Berking et al. 2014). While less information is available on the predictive value of mood repair problems in younger cohorts, rumination, which is a specific maladaptive response, has been well studied. According to a meta-analysis, when baseline depression symptoms are controlled, the tendency to ruminate in response to sadness is a modest prospective predictor of depression among youths (Rood et al. 2009).

However, little is known about whether mood repair can predict course features of a depressive disorder such as recovery from or recurrence of an episode. Among 21 depressed and 19 recovered adults, only one response to sadness, the use of reflection (a presumably adaptive response), predicted recovery from depression 6 months later (Arditte \& Joormann, 2011). Among young adults with remitted depression $(n=99)$, habitual use of MMR (but not adaptive mood repair; AMR) responses predicted a recurrent depression episode across a 3-year follow-up, on average, beyond the prediction offered by prior clinical variables (Kovacs et al. 2009). Thus, the effects of mood repair on the course of a depressive disorder represent an emerging area of research, the scope of which has not yet been extended to juveniles.

Physiological functioning is another domain of individual differences relevant to depression risk. Within this area, the parasympathetic nervous system (PNS) has received particular attention, given its role in emotion experience (e.g. Kreibig, 2010; Levenson, 2014). Respiratory sinus arrhythmia (RSA), the magnitude of heart rate variability (HRV) linked to the respiration cycle, is the most common index of PNS functioning; it reflects the extent of parasympathetic inhibitory input to the heart's pacemaker via the vagal nerve (Thayer et al. 2012). Resting RSA provides information about the ability to adjust physiological arousal to changing internal and external demands and has been regarded as a proxy for overall 'self-regulatory strength' (Geisler et al. 2010): higher values reflect stronger health maintenance capacity. RSA reactivity to challenges usually involves decreased vagal input (vagal withdrawal) which allows increased heart rate, but it also can entail stronger vagal input (vagal augmentation) resulting in decreased heart rate (Berntson et al. 1997). Vagal withdrawal in response to experimental stimuli has been associated with adaptive functioning (Graziano \& Derefinko, 2013), and context-appropriate RSA reactivity is believed to mirror physiological flexibility.

According to several reviews (Rottenberg, 2007; Kemp et al. 2010), depressed (but otherwise healthy) adults generally exhibit lower resting HRV than do controls; this conclusion has been confirmed by some recent studies as well (Brunoni et al. 2013; Liang et al. 2015). According to three separate reports, lower resting RSA levels also characterize diagnosed depressed adolescents as compared with control peers (Tonhajzerova et al. 2009, 2010; Blom et al. 2010), although these studies included only girls. In another sample of adolescents, the null results concerning resting RSA may reflect that the study's definition of recovery probably misclassified some remitted cases as 'currently' depressed (Byrne et al. 2010).

Recent work suggests that RSA may be a prospective predictor of depression symptoms in both adults and youths. Among healthy young adults, resting RSA predicted depression symptoms 1 year later, even after controlling for confounds (Yaptangco et al. 2015). In two partially overlapping samples of young offspring, normative RSA patterns, as well as higher vagal withdrawal in response to an affect trigger, prospectively predicted lower depression symptoms and depression symptom trajectories (Gentzler et al. 2009; Yaroslavsky et al. 2014). However, little is known about whether RSA can predict the clinical course of depressive disorders. In the sole study addressing this issue in adults, vagal withdrawal in response to a sad film predicted recovery from diagnosed depression 6 months later 
(Rottenberg et al. 2005). Thus, while there is increasing evidence of cross-sectional and prospective associations between RSA and depression symptoms, very little is known about the extent to which RSA can inform about the course of depressive disorders across the age span, including adolescence.

Finally, work, which addressed both RSA and mood regulation, suggests that mood repair also may serve as a link between physiological processes and psychological adjustment. For example, among young women at variable risk for depression, normative RSA patterns signaled a reduced likelihood that MMR will lead to depression symptoms (Yaroslavsky et al. 2013a). Emotion regulation also served as a conduit between HRV and various areas of affective functioning (Geisler et al. 2010). However, no study has addressed mood repair as a possible mediator of the relations of RSA and depression recurrence in depression-prone samples.

It is important to examine pathways by which RSA, mood repair and major depressive episode (MDE) recurrence are related in adolescents because clinical depressions that onset very early in the lifespan prognosticate worse functional outcomes across the years than do adult-onset depressions (Lewinsohn et al. 1994; Zisook et al. 2007). Possibly, if recurrent depression early in life can be prevented or forestalled, its long-term negative sequelae may be attenuated. In this regard, it is particularly appropriate to study mood repair and/or RSA as risk factors because these two processes are malleable (e.g. Bhatnagar et al. 2013; Krygier et al. 2013) and therefore can serve as targets in depression prevention.

In light of the above, we tested three hypotheses in our sample of adolescents with prior depression. First, we hypothesized that MMR (but not AMR) predicts recurrent MDEs. Second, we expected that atypical RSA profiles likewise predict recurrent MDEs. And finally, we hypothesized that MMR will mediate the association of atypical RSA profiles and MDE recurrence. To facilitate stringent tests of our hypotheses, mood repair was modeled as a latent construct based on the reports of multiple informants. While our hypotheses build on prior work with adults (Kovacs et al. 2009; Geisler et al. 2010; Yaroslavsky et al. 2013a, b, 2014; Yaptangco et al. 2015), to the best of our knowledge, the current study is the first to model the relations of RSA, mood repair and recurrent major depression in a sample of previously clinically referred adolescents.

\section{Method}

\section{Subjects}

We report on 178 adolescent probands (mean age $=$ 17.14 , S.D. $=1.38$ years), whose histories of MDEs had already been established in a prior genetic investigation in Hungary (e.g. Tamás et al. 2007), but were in remission at the time of their assessment for the present study (time 1; T1). This sample was comprised mostly of males (63\%) and was ethnically representative of the Hungarian population (Caucasian $96 \%$ and $3 \%$ multiethnic, Roma, or other ethnic category). Probands were 9.01 (S.D. $=1.76$ ) years old, on average, at onset of their first MDE, and 3\% $(n=5)$ had had depression episodes in the context of bipolar mood disorder.

Recruitment and diagnostic procedures have been detailed elsewhere (Tamás et al. 2007; Kovacs et al. 2015). Briefly, probands were identified for the prior genetic study across 23 child mental health facilities in Hungary, based on diagnosis [depressive disorder by Diagnostic and Statistical Manual of Mental Disorders, fourth edition (DSM-IV); American Psychiatric Association, 2000], age (7-14 years old at initial screen), medical and cognitive status (free of major systemic medical disorder and intellectual disability), and availability of at least one biological parent and a full biological sibling to participate. Due to funding constraints, only a subset of probands participated in the present study.

\section{Procedures}

T1 visits in the present study included a psychiatric/ psychosocial evaluation and completion of self- and parent-rated questionnaires. As also noted elsewhere (Kovacs et al. 2015), youths also participated in a 1 h-long psychophysiological protocol that probed reactions to various stimuli (presented in counterbalanced order). RSA was continuously monitored via an electrocardiogram (ECG). The stimuli included a 164-s segment from the film 'Champ' (dubbed in Hungarian). This film clip has been extensively used to induce sadness (e.g. Gross \& Levenson, 1995; Rottenberg et al. 2002) and was also pilot tested with Hungarian youths (see Kovacs et al. 2015). We focused on probands' physiological reactions to depictions of sadness because this affect is central to clinical depression. Time 2 (T2) visits involved only a psychiatric/psychosocial evaluation and took place over the subsequent 2 (s.D. $=0.49$ ) years. While the rate of follow-up visits ranged from one to three, $81 \%$ of the youths had two visits. MDE recurrence was based on all information derived across T2 visits.

\section{Determination of depression remission, recurrence and related variables}

Parents and offspring separately completed a DSM-IVbased, semi-structured, psychiatric interview about the adolescent (Interview Schedule for Children and Adolescents: Diagnostic version; ISCA-D) with trained 
clinicians. The ISCA-D has documented reliability (Kiss et al. 2007; Baji et al. 2009): final diagnoses were assigned by consensus among clinicians and were then further verified by pairs of senior diagnosticians. Recovery from an episode of depression was defined as having no more than one clinical symptom and maintaining that essentially asymptomatic state continuously for at least 2 months (Kovacs et al. 1984b). Recurrence was defined as meeting DSM-IV criteria for an episode of MDE after having been essentially symptom free for 2 or more months (Kovacs et al. 1984a).

Parents also provided information about psychotropic medication prescribed for the offspring: those data were coded by type, dose, and start- and enddates. Severity of adolescents' depression symptoms in the prior 2 weeks was quantified via the self-rated Children's Depression Inventory - 2nd edition (CDI; Kovacs \& MHS Staff, 2011).

\section{Mood repair and latent mood repair}

Parents and offspring independently completed the Feelings and My Child, and Feelings and Me (FAM) questionnaires, respectively (Tamás et al. 2007; Gentzler et al. 2009; Bylsma et al. 2016). These questionnaires contain 54 content-wise identical items that quantify the usual extent to which the target youth deploys various AMR and MMR responses to sadness in daily life (e.g. 'when I am sad, I look for a teacher or other adult to talk to'; ' when I am sad, I throw, kick, or hit things', respectively). Responses to items are summed: higher scores reflect greater utilization of adaptive or maladaptive responses. FAM scores have good psychometric properties, including internal consistency, test-retest reliability, and construct, concurrent and predictive validity (Tamás et al. 2007; Kovacs et al. 2009; Bylsma et al. 2015). The maladaptive total score also has been shown to predict depression (Kovacs et al. 2009; Bylsma et al. 2016).

Because we modeled mood repair as a latent construct, we first examined whether latent AMR and MMR factors explain the covariance among parents' and youths' FAM scores. Factor loadings for parents' and youths' FAM scores were made parallel for indicators of the adaptive and maladaptive factor, respectively. Youths' self-reports were free to covary in all models to accommodate shared method variance (see Eid, 2000). The resultant model displayed excellent fit to the data, and was retained for analysis $\left[\chi^{2}(2)=\right.$ 1.07, $p=0.59$, comparative fit index $(\mathrm{CFI})=1.00$, root mean square error of approximation (RMSEA) $=0.00$; $\left.\lambda^{\prime} \mathrm{s}=0.43-0.54, r_{\text {method effect }}=0.26, p<0.01\right]$. Mirroring prior findings (e.g. Yaroslavsky et al. 2013a), the relationship between the AMR and MMR factors was orthogonal $\left(r_{\mathrm{AMR}, \mathrm{MMR}}=-0.02\right)$.

\section{Assessment of RSA}

During the T1 protocol, ECG signals were acquired according to accepted guidelines (Berntson et al. 1997) using $\mathrm{Ag} / \mathrm{AgCl}$ electrodes that were placed in a modified lead II configuration on the subject's chest. Heart values were sampled online at $1000 \mathrm{~Hz}$ using the Mindware Bionex system (MindWare Technologies, Ltd, USA). RSA was calculated using MindWare HRV 3.0.21 software (MindWare Technologies, Ltd, USA). R-wave markers in the ECG signal were processed with the MAD/MED artifact detection algorithm; signals were visually inspected and suspected artifacts were corrected (Berntson et al. 1997). The inter-beat interval (IBI) series was resampled, linearly detrended, and tapered using a Hanning window. HRV was calculated using Fast Fourier transformation analysis of the IBI series, with spectral power values determined in $\mathrm{ms}^{2} / \mathrm{Hz}$ (Berntson et al. 1997). Our index of RSA was the logtransformed high-frequency (HF) power band of HRV (0.15-0.40 Hz range; see Berntson et al. 1997). Hereafter we refer to HF-HRV as RSA, since HFHRV is the power band of HRV that occurs in the typical range of respiration.

We report herein on RSA during a baseline period of paced breathing (180 s), which involved a rising and fading tone to guide subjects' respiration at 12 breaths per min (resting RSA), and during sad mood induction (while watching the film clip described earlier). RSA reactivity was calculated as the difference between resting RSA and RSA during the sad film clip. Thus, positive RSA reactivity values represent vagal withdrawal (decreased RSA during the sad film) while negative values represent vagal augmentation (increased RSA during the sad film). We defined a 'normative RSA profile' as entailing relatively high resting RSA (i.e. at or above the sample's mean) in interaction with RSA withdrawal in response to the sad film clip. This definition is supported by our prior findings (Yaroslavsky et al. 2013b) and the literature on normative characteristics of resting RSA and RSA reactivity to negative affect probes (e.g. Frazier et al. 2004). All other combinations of resting and reactive RSA levels were considered to represent 'atypical' profiles.

\section{Statistical analyses}

Descriptive analyses were conducted via SAS version 9.3 software (SAS Institute, Inc., 2013). The latent variable survival analysis used Mplus version 7.11 software (Muthén \& Muthén, 1998-2012). Latent variable models involved a two-step approach (Anderson \& Gerbing, 1988). First, measurement models were fitted to estimate latent AMR and MMR factors from parent- and offspring-reported FAM scores. Then, 
structural equation models were fitted to estimate MDE recurrence as a function of latent mood repair and RSA patterns.

Robust full information maximum likelihood was used to adjust parameter estimates for missing values (less than $2 \%$ of the sample). Following Hu \& Bentler (1999), non-significant model $\chi^{2}$, along with CFI values of 0.95 or greater, and RMSEA of 0.06 or lower, indicated excellent fit for the measurement model. Fit indices were unavailable for structural models, given that the absence of major depressive disorder recurrence at follow-up assessment was treated as a rightcensured variable. Thus, Wald $\chi^{2}$ tests were used to examine improvement in model fit among nested models.

In all structural models, subject's sex and current psychotropic medication use were co-varied (Barutcu et al. 2005; Rottenberg, 2007; Licht et al. 2008). To account for the fact that subjects were in remission for varying durations at $\mathrm{T} 1$, this variable also was statistically controlled in structural models. Finally, T1 depression symptoms also were statistically controlled, given their probable effects on the risk of MDE recurrence.

\section{Results}

At $\mathrm{T} 1,41 \%$ of the youths already had a history of two or more prior MDEs and $37 \%$ had a lifetime history of psychiatric hospitalization. At the T1 assessment, mean time in remission from the last depression episode was about 5 (S.D. $=2.42$ ) years, while $4.5 \%$ of the youths still had ongoing anxiety disorders. Consistent with a remitted clinical status, the sample's mean CDI score was in the subclinical range (mean=9.26; s.D. $=6.36$; range 0 to 29 ) and only $2 \%$ of the youths were taking prescribed psychotropic medication. Likewise, both self- and parent-rated FAM scores were consistent with remitted depression as the primary clinical status (maladaptive FAM $_{\text {self-rated }}$ mean $=9.57$, S.D. $=6.41$; maladaptive $\mathrm{FAM}_{\text {parent-rated }}$ mean=9.79, s.D. $=7.30$; adaptive $\mathrm{FAM}_{\text {self-rated }}$ mean $=19.38$, S.D. $=8.41$; adaptive $\mathrm{FAM}_{\text {parent-rated }}$ mean $=17.41$, S.D. $=8.33$ ).

Mean baseline resting RSA was 7.24 (S.D. $=1.16$ ), relative to which the sad film clip typically elicited vagal withdrawal $\left(\operatorname{mean}_{\triangle \mathrm{RSA}}=0.73\right.$, S.D. $=0.82, t_{177}=11.88$, $p<0.001$, Cohen's $d=0.90)$. Only 19\% $(n=34)$ of the youths evidenced vagal augmentation during the sad film clip. The 'normative RSA profile', which characterized $44 \%$ of the sample, consisted of the combination of high baseline RSA (i.e. at or above the mean) and RSA withdrawal during sad film viewing. Rates of 'atypical profiles' ranged from $6 \%$ (high resting RSA and RSA augmentation during the sad film) to $37 \%$ (low resting RSA and RSA withdrawal during the sad film).
During the follow-up (mean $=2.08$, S.D. $=0.49$ years), $17 \%(n=30)$ of the youths had an MDE recurrence, with no significant difference in rates between males and females (18.6 and $13.9 \%$, respectively; $p>0.41$ ). Altogether $15.7 \%$ of the sample had interim mental health treatment, including in-patient hospitalization (2\%) and psychotropic medication (6\%). Mental health treatment during the follow-up was significantly more likely among youths with a depression recurrence (43\%), compared with youths who remained in remission $(10 \%)$ (Fisher's exact test $p<0.001)$.

\section{Does mood repair predict a recurrent MDE among adolescents?}

In a structural model, time to the first MDE, with the date of $\mathrm{T} 1$ as the start point, was regressed on the two latent mood repair factors, with subject's sex, current psychotropic medication use and duration of remission at $\mathrm{T} 1$ as covariates $^{1,2}+$. In support of hypothesis 1, T1 MMR predicted the risk of recurrent depression $[b=1.34, p<0.01$, hazard ratio $=3.80,95 \%$ confidence interval (CI) 1.39-10.40]. In contrast, T1 AMR was unrelated to MDE recurrence, suggesting that habitual use of AMR responses does not protect against recurrent depression. Results were unchanged when T1 depression symptoms (CDI scores) were covaried in the model. Thus, reports of MMR at T1 predicted recurrent depression, independent of $\mathrm{T} 1$ depression levels.

\section{Do RSA patterns predict a recurrent MDE among adolescents?}

To test hypothesis 2, we modeled MDE recurrence using baseline RSA and RSA reactivity (main effects) and their interactions (patterns), while controlling for the previously mentioned covariates. Contrary to expectations, neither the main effect of RSA nor the interaction of the two RSA indices predicted depression episode recurrence $\left(b_{\mathrm{RSA}}=0.29, p=0.25 ; b_{\triangle \mathrm{RSA}}\right.$ $\left.=-0.13, p=0.65 ; b_{\mathrm{RSA} \times \Delta \mathrm{RSA}}=-0.55, p=0.15\right)$.

\section{Is mood repair a mediator between RSA patterns and recurrent MDE among adolescents?}

Failure to find a direct association between a predictor (e.g. RSA patterns) and an outcome (e.g. MDE recurrence) has historically been viewed as a contraindication to test for mediation effects. However, according to current perspectives, the aforementioned approach has significant limitations (MacKinnon et al. 2002; Shrout \& Bolger, 2002; Hayes, 2009; Zhao et al. 2010).

t The notes appear after the main text. 


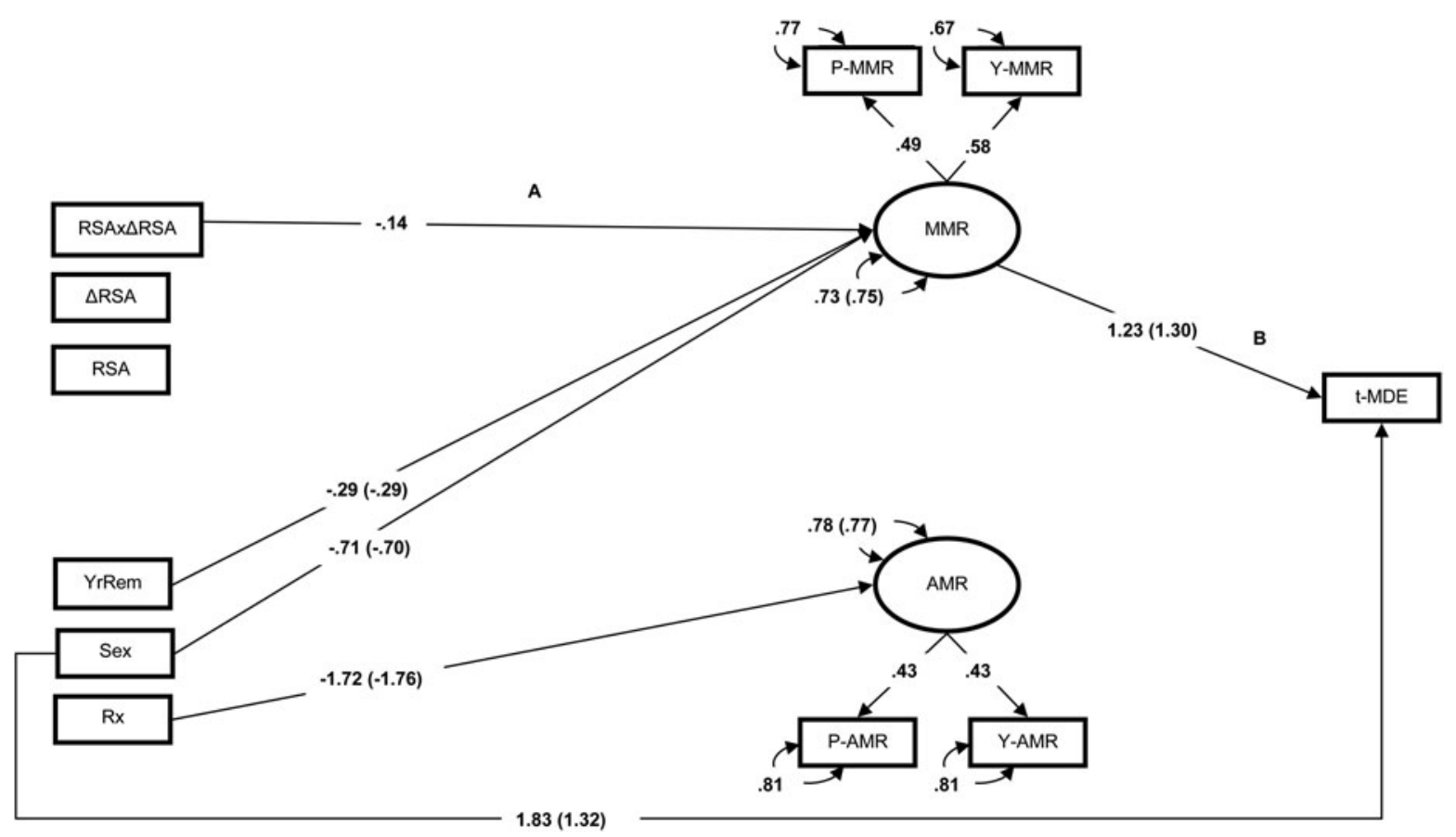

Fig. 1. Standardized first- and second-order structural equation models of latent mood repair mediating the effects of respiratory sinus arrhythmia (RSA) patterns on major depressive episode (MDE) recurrence. Effects of categorical predictors are standardized with respect to the outcome variable. Effects of maladaptive mood repair (MMR) factor on time to episode recurrence are unstandardized. Parameters within parentheses are from the first-order effects models. Parameters outside parentheses are from the second-order effects models. Parameters in bold face are significant $(p<0.05)$. P, Parent report; Y, youth self-report; RSA, RSA during paced breathing; $\triangle \mathrm{RSA}$, change from paced breathing RSA to RSA during the sad film; RSA $\times \triangle$ RSA, second-order (interaction) effects of RSA indices; AMR, adaptive mood repair factor; Rx, prescribed medication use; Sex, high value represents males; YrRem, years in remission at time 1; t-MDE, time-to-episode recurrence by time 2.

Given our hypotheses and previous findings, we therefore examined a mediational model of the relations of RSA patterns, depression recurrence and mood repair. The models included the aforementioned covariates, main and interactive effects of RSA on the two mood repair factors, and the indirect effects of RSA patterns via mood repair on risk of MDE recurrence ${ }^{3}$.

While resting RSA and RSA reactivity (each alone) did not predict the mood repair factors, their interaction (second-order effects) significantly predicted MMR $\left(\beta=-0.14, p<0.05, \Delta R^{2}=0.03\right)$ and significantly improved model fit $\left[\chi^{2}(1)=3.99, p<0.05\right]$. Simple slopes analyses of the interaction revealed that atypical RSA patterns (high resting RSA + augmentation, low resting RSA + withdrawal to the sad film) predicted higher rates of MMR responses $(\beta=0.21)$, whereas normative RSA patterns (high resting RSA + withdrawal to the sad film) predicted lower rates of maladaptive strategy use $(\beta=-0.05)$. The model is depicted in Fig. 1.

To determine whether MMR mediates a potential association between RSA patterns and depression recurrence, regression weights and their respective standard errors (Fig. 1, paths A and B) were submitted to the PRODCLIN program (MacKinnon et al. 2007).
Consistent with hypothesis 3, RSA patterns were linked to MDE recurrence via MMR $\left(b_{M M R, ~ R S A} \times{ }_{\triangle R S A}\right.$ $=-0.17,95 \% \mathrm{CI}-0.13$ to -1.12 ). Adolescents with atypical T1 RSA patterns had more extensive MMR repertoires that, in turn, increased their risk for a recurrent depression episode later on (hazard ratio $=1.19$ ), relative to peers with normative T1 RSA patterns, who displayed less extensive MMR response repertoires (hazard ratio $=0.90$ ). Fig. 2 portrays these results: recurrent MDE risk is modeled since T1, controlling for length of remission at $\mathrm{T} 1$.

\section{Discussion}

In the first study to model the relations of RSA, mood repair and recurrent major depression, we found that MMR response use among previously clinically referred adolescents was a prospective predictor of MDE recurrence over a 2-year period and also served as a conduit through which atypical PNS functioning (RSA patterns) contributed to depression recurrence. Importantly, the predictive value of MMR was above and beyond the prospective prediction offered by prior depression levels (or prior MDEs). In other 


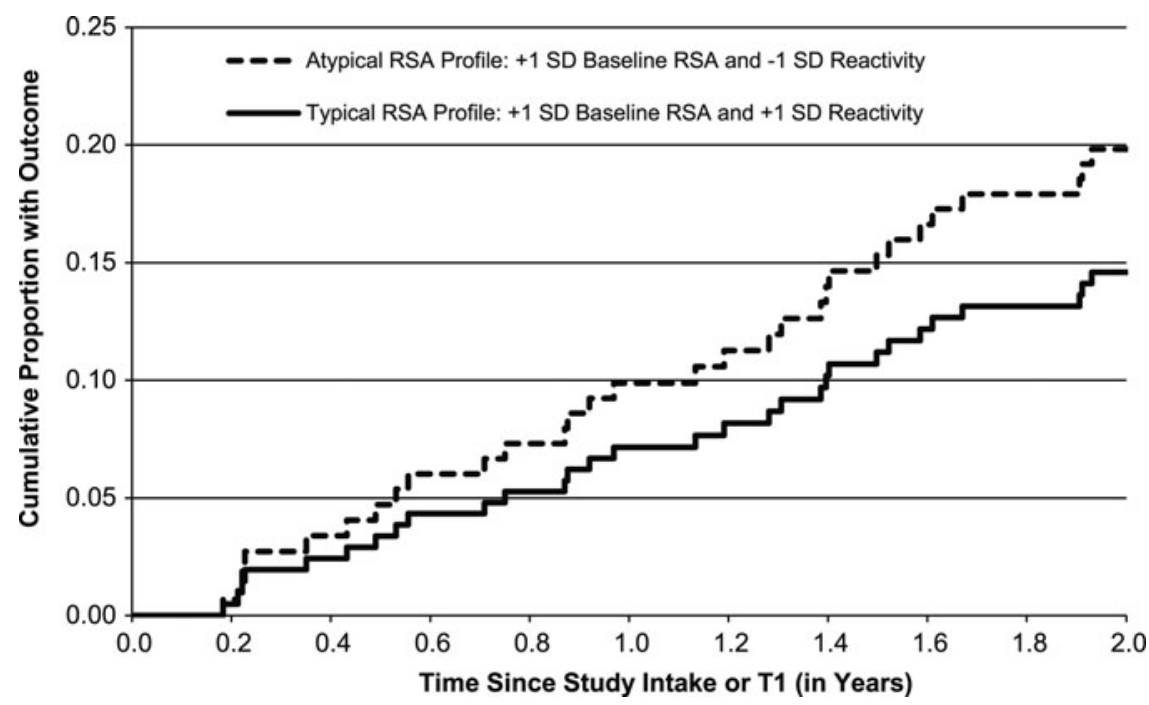

Fig. 2. Estimated effects of atypical and typical (normative) respiratory sinus arrhythmia (RSA) profiles via maladaptive mood repair on the cumulative probability of a recurrent major depression episode in remitted adolescent probands. SD, Standard deviation; T1, time 1.

words, youths who reported habitually responding to sadness in ways that maintained or exacerbated (rather than attenuated) that affect were the ones most likely to have a recurrent depression episode. Notably, even large repertoires of AMR responses did not lower the risk of recurrent depression and thus failed to serve as a protective factor. These results extend to a juvenile cohort our previous finding that MMR is a prognosticator of the clinical course of depressive illness in adults (Kovacs et al. 2009).

Atypical RSA patterns and MMR were significantly associated. And, although we failed to confirm a direct link between RSA and recurrent MDE, the findings supported our third hypothesis that MMR is the conduit through which atypical PNS functioning contributes to depression recurrence. It is of note that combining multiple indexes of PNS functioning (rather than relying on a single index) revealed the contribution of RSA to depression recurrence, a finding also consistent with work on adults (Yaroslavsky et al. 2013b). Because RSA entails a complex and dynamic physiological process, it is logical that taking into account both its tonic (resting) and phasic (reactivity) levels can provide stronger predictive power than can a single index. And yet, even RSA profiles tend to account only for a small proportion of the variance in behavioral outcomes, suggesting that our study was not sufficiently powered to detect its direct association to a relatively low frequency outcome (MDE recurrence).

What is the mechanism whereby mood repair and RSA jointly contribute to recurrent depression? RSA is widely regarded as an index of inhibitory processes that facilitate optimal (flexible) use of physiological resources and response selection, and, hence, a proxy for self-regulatory abilities (Thayer \& Lane, 2000; Porges, 2007). Self-regulatory systems, in turn, have been linked both to attention deployment and affect regulation (Porges, 1992; Thayer \& Lane, 2009). For example, the flexible use of attention is one of the key features of AMR (Kovacs \& Yaroslavsky, 2014), as also documented by laboratory studies of depression-prone adults and adolescents (Joormann et al; 2007; Kovacs et al. 2015). Because mood repair involves multiple response systems, including the PNS, it is likely to be influenced by the adverse effects of suboptimal vagal control on attention deployment. Indeed, the relationship between RSA and attention is well documented (e.g. Park et al. 2012, 2013) and dysfunctional attention processes have been implicated in depression (e.g. Joormann, 2004). Problematic RSA is likely to undermine the flexible use of attention and thereby facilitate maladaptive responses to sadness, which render that affect more enduring or more intense. Such dysphoric affect experiences can become the prelude to an array of related depression symptoms and culminate in an episode of clinical depression.

The present investigation extends the literature on risk factors for recurrent depression by having documented that individual differences in the behavioral-psychological and physiological domains are intertwined as prospective predictors of clinical course. Additionally, to our knowledge, this is the first study to show that a combination of such individual difference variables predicts a recurrent MDE in adolescents. Importantly, the variables we examined are malleable. 
Improving mood repair, or changing the way a person responds to sad affect, has been identified as the central psychotherapeutic target for depressed adults (Berking et al. 2008) and children and adolescents (Kovacs \& Lopez-Duran, 2012), and cognitive approaches to dealing with sadness have long been featured in cognitive-behavior therapy for depression (Beck et al. 1979). Thus, a variety of behavioral, cognitive or interpersonal regulatory strategies could be adapted for use in depression prevention programs. There also are indications that RSA can be modified through meditation (e.g. Nesvold et al. 2011; Bhatnagar et al. 2013; Krygier et al. 2013), which therefore also could have a role in programs to prevent or forestall recurrent depression in youths.

Results of our study should be viewed in the context of several limitations. We acknowledge that our effect sizes have been no larger than medium in magnitude and sometimes were small. However, the risk of a new depression episode, which is an important clinical outcome, is influenced by a large number of factors that include but are not limited to mood repair and RSA, none of which alone would be expected to have a large influence. Indeed, one challenge for future research is to identify combinations of risk factors that provide the best prospective prediction of depression for any given person. Another limitation is that we did not control for respiration in our computation of RSA during sad mood induction, which can influence estimates of PNS activity (e.g. Grossman \& Kollai, 1993). Further, youths were in remission from depression for variable durations at $\mathrm{T} 1$, for which we only could control statistically. Therefore, survival time before a recurrent depression was modeled from study entry, which truncated the true time to recurrence. Finally, the results may not generalize to populations with adult-onset depressive disorders, partly because those groups consist mostly of females. All in all, however, the strengths of the present study outweigh its limitations and point to useful directions in the search to reduce the rate of recurrent depression episodes in youths.

\section{Acknowledgements}

This study was supported by National Institutes of Health grant MH084938 and by Hungarian Scientific Research Fund grant NN85285.

\section{Declaration of Interest}

M.K. receives royalties from Multi-Health Systems Inc. The other authors have no conflicts to declare.

\section{Notes}

${ }^{1}$ To avoid a singularity in the information matrix, the effect of psychotropic medication on time-to-episode recurrence was constrained to 0 value.

${ }^{2}$ We also examined whether an anxiety disorder at $\mathrm{T} 1$ acted as an additional confound. However, in a model that included all the key variables and covariates (sex, medication, duration of remission, RSA profile), T1 anxiety disorder did not predict MMR $(b=1.51$, S.E. $=1.11, p=0.173)$ or MDE recurrence $(b=-0.98$, S.E. $=1.63, p=0.547)$; therefore, it was not considered further in our models.

${ }^{3}$ In response to a request by a reviewer, we re-ran our mediation model using number of prior depressive episodes instead of $\mathrm{T} 1$ depression symptom severity as a covariate. However, the number of prior depressive episodes did not predict key variables (AMR, $b=0.33, p=0.24$; MMR, $b=0.10, p=0.57$; depression recurrence, $b=0.10, p=0.83$ ), nor did it alter our major findings (RSA patterns' effects on MMR, $b=-0.14, p=0.05$; MMR's effects on depression recurrence, $b=1.35, p<0.05$ ).

\section{References}

Aldao A, Nolen-Hoeksema S, Schweizer S (2010). Emotion-regulation strategies across psychopathology: a meta-analytic review. Clinical Psychology Review 30, 217-237.

American Psychiatric Association (2000). Diagnostic and Statistical Manual - Text Revision (DSM-IV-TRim). American Psychiatric Association: Washington, DC.

Anderson JC, Gerbing DW (1988). Structural equation modeling in practice: a review and recommended two-step approach. Psychological Bulletin 103, 411-423.

Arditte KA, Joormann J (2011). Emotion regulation in depression: reflection predicts recovery from a major depressive episode. Cognitive Therapy and Research 35, 536-543.

Baji I, Lopez-Duran NL, Kovacs M, George CJ, Mayer L, Kapornai K, Kiss E, Gadoros J, Vetro A (2009). Age and sex analyses of somatic complaints and symptom presentation of childhood depression in a Hungarian clinical sample. Journal of Clinical Psychiatry 70, 1467-1472.

Barutcu I, Esen AM, Kaya D, Turkmen M, Karakaya O, Melek M, Esen OB, Basaran Y (2005). Cigarette smoking and heart rate variability: dynamic influence of parasympathetic and sympathetic maneuvers. Annals of Noninvasive Electrocardiology 10, 324-329.

Beck AT, Rush AJ, Shaw BF, Emery G (1979). Cognitive Therapy of Depression. The Guilford Press: New York.

Berking M, Wirtz CM, Svaldi J, Hofmann SG (2014). Emotion regulation predicts symptoms of depression over five years. Behaviour Research and Therapy 57, 13-20.

Berking M, Wupperman P, Reichardt A, Pejic T, Dippel A, Znoj H (2008). Emotion-regulation skills as a treatment target in psychotherapy. Behaviour Research and Therapy 46, 1230-1237.

Berntson GG, Bigger Jr. JT, Eckberg DL, Grossman P, Kaufmann PG, Malik M, Nagaraja HN, Porges SW, Saul JP, Stone PH, van der Molen MW (1997). Heart rate 
variability: origins, methods, and interpretive caveats. Psychophysiology 34, 623-648.

Bhatnagar R, Phelps L, Rietz K, Juergens T, Russell D, Miller N, Ahearn E (2013). The effects of mindfulness training on post-traumatic stress disorder symptoms and heart rate variability in combat veterans. Journal of Alternative and Complementary Medicine 19, 860-861.

Blom EH, Olsson EM, Serlachius E, Ericson M, Ingvar M (2010). Heart rate variability (HRV) in adolescent females with anxiety disorders and major depressive disorder. Acta Paediatrica 99, 604-611.

Brunoni AR, Kemp AH, Dantas EM, Goulart AC, Nunes MA, Boggio PS, Mill JG, Lotufo PA, Fregni F, Benseñor IM (2013). Heart rate variability is a trait marker of major depressive disorder: evidence from the sertraline vs. electric current therapy to treat depression clinical study. International Journal of Neuropsychopharmacology 16, 1937-1949.

Burcusa SL, Iacono WG (2007). Risk for recurrence in depression. Clinical Psychology Review 27, 959-985.

Bylsma LM, Yaroslavsky I, Rottenberg J, Kiss E, Kapornai K, Halas K, Dochnal R, Lefkovics E, Baji I, Vetró A, Kovacs M (2016). Familiality of mood repair responses among youth with and without histories of depression. Cognition and Emotion 30, 807-816.

Byrne ML, Sheeber L, Simmons JG, Davis B, Shortt JW, Katz LF, Allen NB (2010). Autonomic cardiac control in depressed adolescents. Depression and Anxiety 27, 10501056.

Cuthbert BN, Insel TR (2013). Toward the future of psychiatric diagnosis: the seven pillars of RDoC. BMC Medicine 11, 126.

Ehring T, Fischer S, Schnulle J, Bosterling A, TuschenCaffier B (2008). Characteristics of emotion regulation in recovered depressed versus never depressed individuals. Personality and Individual Differences 44, 1574-1584.

Eid M (2000). A multitrait-multimethod model with minimal assumptions. Psychometrika 65, 241-261.

Farb NA, Irving JA, Anderson AK, Segal ZV (2015). A two-factor model of relapse/recurrence vulnerability in unipolar depression. Journal of Abnormal Psychology 124, 38-53.

Frazier TW, Strauss ME, Steinhauer SR (2004). Respiratory sinus arrhythmia as an index of emotional response in young adults. Psychophysiology 41, 75-83.

Geisler FC, Vennewald N, Kubiak T, Weber H (2010). The impact of heart rate variability on subjective well-being is mediated by emotion regulation. Personality and Individual Differences 49, 723-728.

Gentzler AL, Santucci AK, Kovacs M, Fox NA (2009). Respiratory sinus arrhythmia reactivity predicts emotion regulation and depressive symptoms in at-risk and control children. Biological Psychology 82, 156-163.

Graziano P, Derefinko K (2013). Cardiac vagal control and children's adaptive functioning: a meta-analysis. Biological Psychology 94, 22-37.

Gross JJ (2013). Emotion regulation: taking stock and moving forward. Emotion 13, 359-365.

Gross JJ, Levenson RW (1995). Emotion elicitation using films. Cognition and Emotion 9, 87-108.
Grossman P, Kollai M (1993). Respiratory sinus arrhythmia, cardiac vagal tone, and respiration: within- and betweenindividual relations. Psychophysiology 30, 486-495.

Hardeveld F, Spijker J, De Graaf R, Nolen WA, Beekman ATF (2010). Prevalence and predictors of recurrence of major depressive disorder in the adult population. Acta Psychiatrica Scandinavica 122, 184-191.

Hardeveld F, Spijker J, De Graaf R, Nolen WA, Beekman ATF (2013). Recurrence of major depressive disorder and its predictors in the general population: results from The Netherlands Mental Health Survey and Incidence Study (NEMESIS). Psychological Medicine 43, 39-48.

Harkness KL, Theriault JE, Stewart JG, Bagby RM (2014). Acute and chronic stress exposure predicts 1-year recurrence in adult outpatients with residual depression symptoms following response to treatment. Depression and Anxiety 31, 1-8.

Hayes AF (2009). Beyond Baron and Kenny: statistical mediation analysis in the new millennium. Communication Monographs 76, 408-420.

Hu LT, Bentler PM (1999). Cutoff criteria for fit indexes in covariance structure analysis: conventional criteria versus new alternatives. Structural Equation Modeling: A Multidisciplinary Journal 6, 1-55.

Joormann J (2004). Attentional bias in dysphoria: the role of inhibitory processes. Cognition and Emotion 18, 125-147.

Joormann J, Siemer M, Gotlib IH (2007). Mood regulation in depression: differential effects of distraction and recall of happy memories on sad mood. Journal of Abnormal Psychology 116, 484-490.

Kanske P, Heissler J, Schonfelder S, Wessa M (2012). Neural correlates of emotion regulation deficits in remitted depression: the influence of regulation strategy, habitual regulation use, and emotional valence. NeuroImage 61, 686-693.

Kemp AH, Quintana DS, Gray MA, Felmingham KL, Brown K, Gatt JM (2010). Impact of depression and antidepressant treatment on heart rate variability: a review and metaanalysis. Biological Psychiatry 67, 1067-1074.

Kiss E, Gentzler AM, George C, Kapornai K, Tamás Z, Kovacs M, Vetró Á (2007). Factors influencing motherchild reports of depressive symptoms and agreement among clinically referred depressed youngsters in Hungary. Journal of Affective Disorders 100, 143-151.

Kovacs M, Feinberg TL, Crouse-Novak M, Paulauskas SL, Pollock M, Finkelstein R (1984a). Depressive disorders in childhood. II. A longitudinal study of the risk for a subsequent major depression. Archives of General Psychiatry 41, 643-649.

Kovacs M, Feinberg TL, Crouse-Novak MA, Paulauskas SL, Finkelstein R (1984b). Depressive disorders in childhood. I. A longitudinal prospective study of characteristics and recovery. Archives of General Psychiatry 41, 229-237.

Kovacs M, Lopez-Duran NL (2012). Contextual emotion regulation therapy: a developmentally-based intervention for pediatric depression. Child and Adolescent Psychiatric Clinics of North America 21, 327-343. 
Kovacs M, MHS Staff (2011). Children's Depression Inventory 2 (CDI 2), 2nd edn. Technical manual. Multi-Health Systems Inc.: North Tonawanda, NY.

Kovacs M, Rottenberg J, George C (2009). Maladaptive mood repair responses distinguish young adults with early-onset depressive disorders and predict future depression outcomes. Psychological Medicine 39, 1841-1854.

Kovacs M, Yaroslavsky I (2014). Practitioner review: dysphoria and its regulation in child and adolescent depression. Journal of Child Psychology and Psychiatry 55, 741-757.

Kovacs M, Yaroslavsky I, Rottenberg J, George CJ, Baji I, Benák I, Dochnal R, Halas K, Kiss E, Vetró Á, Kapornai K (2015). Mood repair via attention refocusing or recall of positive autobiographical memories by adolescents with pediatric-onset major depression. Journal of Child Psychology and Psychiatry 56, 1108-1117.

Kreibig SD (2010). Autonomic nervous system activity in emotion: a review. Biological Psychology 84, 394-421.

Krygier JR, Heathers JA, Shahrestani S, Abbott M, Gross JJ, Kemp AH (2013). Mindfulness meditation, well-being, and heart rate variability: a preliminary investigation into the impact of intensive Vipassana meditation. International Journal of Psychophysiology 89, 305-313.

Levenson RW (2014). The autonomic nervous system and emotion. Emotion Review 6, 100-112.

Lewinsohn PM, Clarke GN, Seeley JR, Rohde P (1994). Major depression in community adolescents: age at onset, episode duration, and time to recurrence. Journal of the American Academy of Child and Adolescent Psychiatry 33, 809-818.

Liang CS, Lee JF, Chen CC, Chang YC (2015). Reactive heart rate variability in male patients with first-episode major depressive disorder. Progress in Neuro-Psychopharmacology and Biological Psychiatry 56, 52-57.

Licht CM, de Geus EJ, Zitman FG, Hoogendijk WJ, van Dyck R, Penninx BW (2008). Association between major depressive disorder and heart rate variability in the Netherlands Study of Depression and Anxiety (NESDA). Archives of General Psychiatry 65, 1358-1367.

MacKinnon DP, Fritz MS, Williams J, Lockwood CM (2007). Distribution of the product confidence limits for the indirect effect: program PRODCLIN. Behavior Research Methods 39, 384-389.

MacKinnon DP, Lockwood CM, Hoffman JM, West SG, Sheets V (2002). A comparison of methods to test mediation and other intervening variable effects. Psychological Methods 7, 83-104.

Marchetti I, Koster EH, Sonuga-Barke EJ, De Raedt R (2012). The default mode network and recurrent depression: a neurobiological model of cognitive risk factors. Neuropsychology Review 22, 229-251.

Mueller TI, Leon AC, Keller MB, Solomon DA, Endicott J, Coryell W, Warshaw M, Maser JD (1999). Recurrence after recovery from major depressive disorder during 15 years of observational follow-up. American Journal of Psychiatry 156, 1000-1006.

Muthén LK, Muthén BO (1998-2012). Mplus User's Guide, 7th edn. Muthén \& Muthén: Los Angeles, CA.

Nesvold A, Fagerland MW, Davanger S, Ellingsen Ø, Solberg EE, Holen A, Sevre K, Atar D (2011). Increased heart rate variability during nondirective meditation. European Journal of Preventive Cardiology 19, 773-780.

Nolen-Hoeksema S, Morrow J (1991). A prospective study of depression and posttraumatic stress symptoms after a natural disaster: the 1989 Loma Prieta earthquake. Journal of Personality and Social Psychology 61, 115-121.

Park G, Van Bavel JJ, Vasey MW, Thayer JF (2012). Cardiac vagal tone predicts inhibited attention to fearful faces. Emotion 12, 1292-1302.

Park G, Vasey MW, Van Bavel JJ, Thayer JF (2013). Cardiac vagal tone is correlated with selective attention to neutral distractors under load. Psychophysiology 50, 398-406.

Porges SW (1992). Autonomic regulation and attention. In Attention and Information Processing in Infants and Adults (ed. B. A. Campbell, H. Hayne and R. Richardson), pp. 201-223. Lawrence Erlbaum Associates, Inc.: Hillside, NJ.

Porges SW (2007). The polyvagal perspective. Biological Psychology 74, 116-143.

Rao U, Chen LA (2009). Characteristics, correlates, and outcomes of childhood and adolescent depressive disorders. Dialogues in Clinical Neuroscience 11, 45-62.

Rood L, Roelofs J, Bögels SM, Nolen-Hoeksema S, Schouten E (2009). The influence of emotion-focused rumination and distraction on depressive symptoms in nonclinical youth: a meta-analytic review. Clinical Psychology Review 29, 607-616.

Rottenberg J (2007). Cardiac vagal control in depression: a critical analysis. Biological Psychology 74, 200-211.

Rottenberg J, Gross JJ, Wilhelm FH, Najmi S, Gotlib IH (2002). Crying threshold and intensity in major depressive disorder. Journal of Abnormal Psychology 111, 302-312.

Rottenberg J, Salomon K, Gross JJ, Gotlib IH (2005). Vagal withdrawal to a sad film predicts subsequent recovery from depression. Psychophysiology 42, 277-281.

SAS Institute, Inc. (2013). SAS® 9.3 System Options: Reference, 2nd edn. SAS Institute, Inc.: Cary, NC.

Shrout PE, Bolger N (2002). Mediation in experimental and nonexperimental studies: new procedures and recommendations. Psychological Methods 7, 422-445.

Solomon DA, Keller MB, Leon AC, Mueller TI, Lavori PW, Shea MT, Coryell W, Warshaw M, Turvey C, Maser JD, Endicott J (2000). Multiple recurrences of major depressive disorder. American Journal of Psychiatry 157, 229-233.

Tamás Z, Kovacs M, Gentzler AL, Tepper P, Gádoros J, Kiss E, Kapornai K, Vetró Á (2007). The relations of temperament and emotion self-regulation with suicidal behaviors in a clinical sample of depressed children in Hungary. Journal of Abnormal Child Psychology 35, 640-652.

Thayer JF, Åhs F, Fredrikson M, Sollers JJ, Wager TD (2012). A meta-analysis of heart rate variability and neuroimaging studies: implications for heart rate variability as a marker of stress and health. Neuroscience and Biobehavioral Reviews 36, 747-756.

Thayer JF, Lane RD (2000). A model of neurovisceral integration in emotion regulation and dysregulation. Journal of Affective Disorders 61, 201-216.

Thayer JF, Lane RD (2009). Claude Bernard and the heartbrain connection: further elaboration of a model of 
neurovisceral integration. Neuroscience and Biobehavioral Reviews 33, 81-88.

Tonhajzerova I, Ondrejka I, Javorka K, Turianikova Z, Farsky I, Javorka M (2010). Cardiac autonomic regulation is impaired in girls with major depression. Progress in NeuroPsychopharmacology and Biological Psychiatry 34, 613-618.

Tonhajzerova I, Ondrejka I, Javorka M, Adamik P, Turianikova Z, Kerna V, Javorka K, Calkovska A (2009). Respiratory sinus arrhythmia is reduced in adolescent major depressive disorder. European Journal of Medical Research 14, 280-283.

Yaptangco M, Crowell SE, Baucom BR, Bride DL, Hansen EJ (2015). Examining the relation between respiratory sinus arrhythmia and depressive symptoms in emerging adults: a longitudinal study. Biological Psychology 110, 34-41.

Yaroslavsky I, Bylsma LM, Rottenberg J, Kovacs M (2013a). Combinations of resting RSA and RSA reactivity impact maladaptive mood repair and depression symptoms. Biological Psychology 94, 272-281.

Yaroslavsky I, Rottenberg J, Kovacs M (2013b). The utility of combining RSA indices in depression prediction. Journal of Abnormal Psychology 122, 314-321.

Yaroslavsky I, Rottenberg J, Kovacs M (2014). Atypical patterns of respiratory sinus arrhythmia index an endophenotype for depression. Development and Psychopathology 26, 1337-1352.

Zhao X, Lynch JG, Chen Q (2010). Reconsidering Baron and Kenny: myths and truths about mediation analysis. Journal of Consumer Research 37, 197-206.

Zisook S, Lesser I, Stewart JW, Wisniewski SR, Balasubramani GK, Fava M, Gilmer WS, Dresselhaus TR, Thase ME, Nierenberg AA, Trivedi MH, Rush AJ (2007). Effect of age at onset on the course of major depressive disorder. American Journal of Psychiatry 164, 1539-1546. 